\title{
Evaluation of Osteocalcin Levels in Saliva of Periodontitis Patients and Their Correlation with the Disease Severity: A Cross-Sectional Study
}

\author{
Hamda Shazam ${ }^{1}$ Fouzia Shaikh² Zaheer Hussain ${ }^{3}$ \\ Zohaib Khurshid ${ }^{6, \odot}$ \\ ${ }^{1}$ Department of Oral Pathology, College of Dentistry, Ziauddin \\ University, Karachi, Pakistan \\ 2Department of Pathology, Ziauddin University, Karachi, Pakistan \\ ${ }^{3}$ Department of Periodontology, Altamash Institute of Dental \\ Medicine, Karachi, Pakistan \\ ${ }^{4}$ Department of Oral Biology, Altamash Institute of Dental \\ Medicine, Karachi, Pakistan \\ ${ }^{5}$ Department of Oral Pathology, College of Dentistry, Ziauddin \\ University, Karachi, Pakistan \\ 6 Prosthodontics and Implantology, College of Dentistry, King Faisal \\ University, Al Ahsa, Kingdom of Saudi Arabia
}

M. Mansoor Majeed ${ }^{4}$ Saba Khan ${ }^{5}$

Address for correspondence Hamda Shazam, BDS, Department of Oral Pathology, College of Dentistry, Ziauddin University, Karachi 75600, Pakistan (e-mail: hamdashazam@gmail.com).

Eur J Dent:2020;14:352-359

\begin{abstract}
Keywords

- saliva

- osteocalcin

- periodontitis

- salivary biomarker

Objectives The present study aimed to investigate osteocalcin levels in saliva of healthy and periodontitis patients and correlate these levels with periodontitis severity.

Materials and Methods This cross-sectional study was conducted in a hospital setup. A total of 95 individuals participated in the study with 46 subjects in group I (healthy individuals) and 49 subjects in group II (mild, moderate, and severe chronic periodontitis patients). A detailed assessment of clinical periodontal parameters and alveolar bone loss was made. Unstimulated saliva samples were collected from all study subjects and osteocalcin levels were quantitatively analyzed by sandwich enzyme-linked immunosorbent essay technique.

Statistical Analysis One-way analysis of variance, Spearman's correlation test, and Pearson's chi-squared test were applied at a significance level of $95 \%$. $p$-Values less than 0.05 were considered statistically significant.

Results The results showed a significant association of qualification with group II $(p<0.02)$. Bone loss scores were also significantly associated with periodontitis severity $(p<0.01)$. However, no statistically significant difference was observed between group I and group II in terms of mean salivary osteocalcin levels ( $p=0.68$ ). Also, an insignificant correlation was also observed between osteocalcin levels and periodontitis severity $(p=0.13)$.

Conclusion The overall study results showed that there was no significant difference between saliva osteocalcin levels of healthy and periodontitis patients. Also, there was a nonsignificant correlation between osteocalcin levels and periodontitis severity. The findings of the present study support the hypothesis that low osteocalcin levels in saliva might be considered as a poor indicator of periodontal disease progression and severity.
\end{abstract}

DOI https://doi.org/ $10.1055 / \mathrm{s}-0040-1710143$ ISSN 1305-7456.
License terms

(1) (1) $\odot \circledast$ 


\section{Introduction}

Periodontitis is an irreversible chronic oral inflammatory disease leading to progressive destruction of soft and hard tooth-supporting structures. Excessive bony destruction, concomitant loss of periodontal ligament and cementum attachment, the formation of deepened periodontal pockets, frequent tooth mobility, and ultimate tooth loss are all hallmarks of periodontitis. ${ }^{1}$ Besides this, the periodontitis-affected individuals encounter various dental anomalies like multiple tooth loss, edentulism, and masticatory dysfunction, which adversely affect their health and impose negative socioeconomic impacts. ${ }^{2}$ Periodontitis is implicated as a major risk factor for several systemic diseases such as rheumatoid arthritis, heart diseases, and pulmonary infections. ${ }^{3}$

The etiological cause of the periodontal disease is multifactorial, with bacteria as the prime causative agent. ${ }^{4}$ Apart from bacteria, certain intrinsic and extrinsic risk factors also contribute to etiological factors. These include poor oral hygiene habits, restorative dental procedures, immunodeficiency disorders, hematological disorders, nutritional deficiencies, and certain endocrinal abnormalities. ${ }^{5}$

The pathogenesis of periodontitis is complex and involves a cascade of sequential events taking place between the host immune system and bacterial pathogens. On exposure to the toxic by-products and lipopolysaccharides released by microbes, activation of macrophages and monocytes occurs which leads to an influx of certain proinflammatory mediators including cytokines/chemokine (interleukins tumor necrosis factor alpha), prostaglandins, and matrix metalloproteinases. These inflammatory mediators elicit progression of an initial inflammatory lesion to advanced lesion, thereby destroying gingival tissues and underlying alveolar bone. ${ }^{6}$

The prevalence of periodontitis varies globally in terms of severity and progression. According to the recent epidemiological studies, an estimated 9 to $15 \%$ of the adult population worldwide is affected by chronic periodontitis. ${ }^{7}$ Moreover, it is ranked as the sixth most prevalent oral disease. ${ }^{8}$ In Asian countries, its prevalence is reported to be as high as 15 to $20 \%^{9}$ and in Pakistan, the prevalence of severe periodontitis is calculated up to $34.1 \%{ }^{10}$

Human saliva is composed of salivary grand secretions (major/minor), immunoglobulins, peptidases, mucins, hormones, DNA or RNA molecules, and a variety of electrolytes that reflect changes in the physiological and pathological states of the human body. Therefore, saliva is considered as the "mirror" of the body. Saliva owes great benefits as ideal diagnostic medium due to its dynamic properties such as easy-to-approach, noninvasive sampling, cost-effectiveness, and minimal risk of cross-infection. ${ }^{11}$ Nowadays, the role of saliva as liquid biopsy tool has become the cornerstone in point-of-care diagnostic technology that facilitates patient's as well as researchers with the rapid screening and reliable diagnosis of the disease using small portable microchips as detection devices. ${ }^{12,13}$ Since past few years, notable studies have reported that multiple biomarkers can be harvested from human saliva for the earlier diagnosis of oral diseases such as dental caries, periodontitis, oral cancer, oral submucous fibrosis, and oral leukoplakia. Likewise, salivary biomarkers relevant to systemic diseases are also detected such as cardiovascular diseases, diabetes, breast cancer, dengue virus, HIV, human herpes virus-8, and Zika virus. ${ }^{14,15}$ By definition, biomarkers are objectively measured variables that are found in blood serum, saliva, or any other body fluids and act as indicator of normal physiological processes, pathological processes, or responses to therapeutic intervention..$^{16}$ The increased prevalence of periodontitis and inadequate conventional diagnostic methods has paved way for the discovery of salivary biomarkers that prove to be potential diagnostic, prognostic, and disease monitoring markers. ${ }^{17}$

Biomarkers associated with alveolar bone turnover were discussed in a substantial number of studies. ${ }^{18,19}$ Osteocalcin, also known as Gla-protein with a low molecular-weight and high affinity for calcium, is the most abundant nonmineralized bone protein. It occurs in two physiological states: carboxylated and uncarboxylated forms. Carboxylated form of osteocalcin is inactive and present in bone matrix. However, the uncarboxylated form is active state freely present in serum/plasma. Osteocalcin is mainly synthesized and secreted by osteoblasts, odontoblasts, and chondrocytes. ${ }^{20}$ It plays an important role in bone remodeling and is generally considered as bone formation biomarker but in periodontitis where bone hemostasis is perturbed due to higher resorption rate osteocalcin recruits osteoclasts to the site of bone degradation and promotes their differentiation into active osteoclasts. Raised levels of osteocalcin in body fluids point out to abnormal bone turnover activity occurring during periodontitis. Hence, it is now widely accepted as a marker of bone turnover rather than bone formation. ${ }^{21}$

Because of contradictory data available in the literature, the role of osteocalcin in periodontitis is yet not clear. To the best of our knowledge, there has been no study conducted in Pakistan, to estimate osteocalcin levels in periodontitis patients. Hence, in this study, we aimed to investigate osteocalcin levels in saliva of healthy and periodontitis patients and further assess its correlation with periodontitis severity.

\section{Materials and Methods}

This cross-sectional study was conducted at the Multidisciplinary Research laboratory of Ziauddin University in Karachi, Pakistan, with a duration of five months.

The Ethics Review Committee of Ziauddin University approved the study design. (Reference Code: 0950319HSOM)

\section{Sample Size Estimation}

The sample size calculation was done using online sample size calculator of OPENEPI version 3.01 (https://www. openepi.com/SampleSize/SSPropor.htm). After considering $15 \%$ prevalence of periodontitis in Pakistani population from the reference article, ${ }^{22}$ and inserting $7.2 \%$ margin of error at $95 \%$ confidence interval and $80 \%$ power of the study, a sample 
size of total 95 participants was estimated out of which 46 participants were taken in group I (healthy individuals) and 49 participants in group II (mild, moderate, and severe chronic periodontitis patients).

All the study subjects were recruited from the Outpatient Department of Periodontology, Ziauddin College of Dentistry. Before the enrollment of study, signed consent form (bilingual) was attained by each participant describing the nature and duration of the study.

A detailed questionnaire was filled about the brief dental history and demographic characteristics of each participant. Each participant had comprehensive clinical and radiographic examination by the principal investigator. Sample selection followed strict inclusion and exclusion criteria.

\section{Inclusion Criteria}

1. Healthy individuals (both gender) older than 18 years of age, without any periodontal, systemic, or bone disorders. All had minimum 16 number of teeth in their mouth without any previous dental treatment.

2. Chronic periodontitis patients (both gender) older than 18 years of age, with clinical and radiographic features of periodontitis. The chronic periodontitis patients were grouped into mild, moderate, and severe categories based on the classification system proposed by Caton et al. ${ }^{23}$

3. Individuals who were willing to participate in the study.

\section{Exclusion Criteria}

1. Smokers, betel nut users, and tobacco chewers.

2. Pregnant and lactating females.

3. Patients using antibiotics, vitamin D, or calcium supplements for the last 3 months.

4. Medically compromised patients with genetic abnormalities, systemic, or bone disorders.

5. Patients who had undergone any periodontal treatment within the last 6 months.

\section{Clinical Periodontal Examination}

Four clinical periodontal parameters were assessed, including bleeding on probing (BOP), periodontal pocket depth (PPD), plaque index (PI), and clinical attachment level (CAL). Six locations per tooth were examined including buccal, mesiobuccal, distobuccal, mesiolingual, lingual, and distolingual. Third molars were not included in the clinical assessment. All the measurements were recorded by University of North Carolina PCP-15 periodontal probe. A calibrated examiner performed the comprehensive periodontal examination. The mild periodontitis group exhibited CAL 1 to $2 \mathrm{~mm}$, PPD $\leq 4 \mathrm{~mm}$, and $\leq 30 \%$ of radiographic bone loss (RBL). Moderate periodontitis group had CAL 3 to $4 \mathrm{~mm}$, maximum probing depth $\leq 5 \mathrm{~mm}$, and 30 to $50 \%$ of RBL. However, in case of severe periodontitis $\geq 5 \mathrm{~mm}$ of CAL and $\geq 6 \mathrm{~mm}$ of PPD was recorded with RBL extending to middle third of root and beyond. BOP was present in all forms of periodontitis.

\section{Radiographic Assessment}

Alveolar bone loss scores were recorded using Schei ruler and full mouth panoramic radiographs were obtained. Third molars were excluded from the analysis. A marking of $1 \mathrm{~mm}$ thickness represented the distance between cementenamel junction (CEJ) and the alveolar bone crest in normal individuals without bone loss. The bone loss was expressed as a percentage of original bone level, starting $1 \mathrm{~mm}$ below CEJ. Mild periodontitis included bone loss less than one third and in case of moderate periodontitis one-third to one half of alveolar bone loss was observed, whereas severe periodontitis demonstrated $\geq$ one half of alveolar bone loss. No alveolar bone loss was seen in healthy individuals.

\section{Saliva Collection Procedure}

Unstimulated whole saliva was preferably taken since minimal dilution of constituents and larger sample size without any external stimuli can be achieved. Passive drooling method was opted for the collection of unstimulated whole saliva. Initially, all subjects were refrained from performing any dental hygiene procedures like flossing, brushing, chewing for at least 1 hour. Subjects were then asked to "deburr" (let the saliva drip down) by leaning their heads forward and keeping mouths wide open into sterile polypropylene tubes kept on ice. ${ }^{24}$ Approximately $5 \mathrm{~mL}$ of saliva was collected in each tube. Centrifugation at 2,600 g for 15 minutes at $4^{\circ} \mathrm{C}$ was done to avoid any debris contamination. Aliquots of saliva samples were prepared and the combination of protease inhibitor was incorporated to impede degradation of proteins. The supernatant saliva samples were stored at $-80^{\circ} \mathrm{C}$ for subsequent analysis.

\section{Quantitative Analysis of Osteocalcin in Saliva}

Osteocalcin levels in saliva of both groups were analyzed by enzyme-linked immunosorbent essay (ELISA) technique using kits of Cloud-Clone Corp (ELISA kit; Katy, Texas, United States). The procedures were performed as per manual instructions. The minimal detectable dose of osteocalcin was $0.264 \mathrm{ng} / \mathrm{mL}$. The ELISA kit included 96 microplate wells coated with monoclonal osteocalcin-specific antibody. The samples containing antigen were then inserted into each well and bound to biotin-conjugated antibody. Then avidinhorseradish peroxidase complex was also added to the biotin conjugate complex. 3,3',5,5'-Tetramethylbenzidine substrate solution was finally added that detected the antigen by the change in color. The difference in color intensity was measured at $450 \pm 10 \mathrm{~nm}$. A standard curve was plotted between optical density (OD) and the known concentration of each standard solution. The osteocalcin concentrations present in the saliva samples were then calculated by comparing the OD of the samples to the standard curve and the results were expressed in units of $(\mathrm{ng} / \mu \mathrm{L})$.

\section{Statistical Analysis}

All the statistical data was collected, stored, and analyzed using IBM-SPSS version 23.0 (IBM SPSS Statistics for Windows, version 23.0; IBM Corp, Armonk, New York, United 
States). $p$-Values less than 0.05 were considered as statistically significant for analysis. Mean and the standard deviation were determined for quantitative data, whereas qualitative data were expressed as frequencies and percentages. Pearson chi-squared test was applied to assess the association of qualitative variables, whereas one-way analysis of variance (ANOVA) was performed to analyze and compare the mean difference between levels of osteocalcin and clinical parameters in both groups. Spearman rank correlation test was done to estimate the correlation of saliva osteocalcin levels with periodontitis and other variables. Pie chart and bar diagrams were also drawn to give a graphical presentation of the data.

\section{Results}

- Table 1 illustrates the sociodemographic characteristics of the study population. A total of 95 participants were divided into two study groups including 46 healthy individuals in group I and 49 chronic periodontitis patients in group II ( - Fig. 1). In group II, $57.1 \%$ of samples were found with a mean age of $33.40 \pm 8.05$ years. About $69.4 \%$ were males and married and $32.7 \%$ were graduates. Among group I, $50 \%$ of samples had a mean age of $31.54 \pm 6.20$ years, $58.7 \%$ were male, $57.8 \%$ were married, and $58.7 \%$ were undergraduate. There was no statistically significant difference between the two study groups in terms of age, gender, and marital status with the $p$-values $0.48,0.27$, and 0.24 , respectively. However, the results of the Pearson chi-squared test showed a significant association of qualification $(p<0.02)$ within group II (-Fig. 2).

- Table 2 represents the mean differences between the osteocalcin concentrations and the clinical periodontal parameters of both study groups using ANOVA. Results depicted no significant differences between the mean values of osteocalcin concentrations in normal, mild, moderate, and severe periodontitis group $(p=0.68)$; however, a

Table 1 Details of demographic characteristics of study subjects

\begin{tabular}{|c|c|c|c|c|c|c|}
\hline \multicolumn{2}{|c|}{ Characteristics } & \multicolumn{4}{|c|}{ Group } & \multirow[t]{3}{*}{$p$-Value } \\
\hline & & \multicolumn{2}{|c|}{$\begin{array}{ll} & \text { Healthy } \\
(n=46) & \end{array}$} & \multicolumn{2}{|c|}{$\begin{array}{l}\text { Periodontitis } \\
(n=49)\end{array}$} & \\
\hline & & $N$ & $\%$ & $N$ & $\%$ & \\
\hline \multirow[t]{3}{*}{ Age group } & $20-30$ y & 23 & 50.0 & 21 & 42.9 & \multirow[t]{3}{*}{0.48} \\
\hline & $31-45 y$ & 23 & 50.0 & 28 & 57.1 & \\
\hline & Mean \pm SD & 31.54 & \pm 6.20 & 33.40 & \pm 8.05 & \\
\hline \multirow[t]{2}{*}{ Gender } & Male & 27 & 58.7 & 34 & 69.4 & \multirow[t]{2}{*}{0.27} \\
\hline & Female & 19 & 41.3 & 15 & 30.6 & \\
\hline \multirow[t]{2}{*}{ Marital status } & Married & 26 & 57.8 & 34 & 69.4 & \multirow[t]{2}{*}{0.24} \\
\hline & Unmarried & 19 & 42.2 & 15 & 30.6 & \\
\hline \multirow[t]{5}{*}{ Qualification } & Below 10th grade & 2 & 4.3 & 7 & 14.3 & \multirow[t]{5}{*}{$0.02^{\mathrm{a}}$} \\
\hline & Below 12th grade & 5 & 10.9 & 11 & 22.4 & \\
\hline & Undergraduate & 7 & 15.2 & 13 & 26.5 & \\
\hline & Graduate & 27 & 58.7 & 16 & 32.7 & \\
\hline & Postgraduate & 5 & 10.9 & 2 & 4.1 & \\
\hline
\end{tabular}

Abbreviation: SD, standard deviation.

${ }^{a} p$-Value $<0.05$ was considered significant using Pearson chi-squared test.
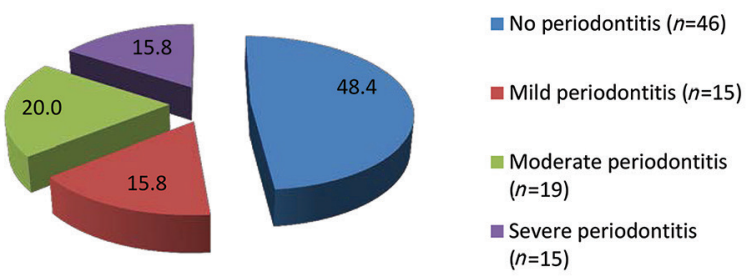

Fig. 1 Pie chart representing sample size distribution for healthy and periodontitis groups $(n=95)$.

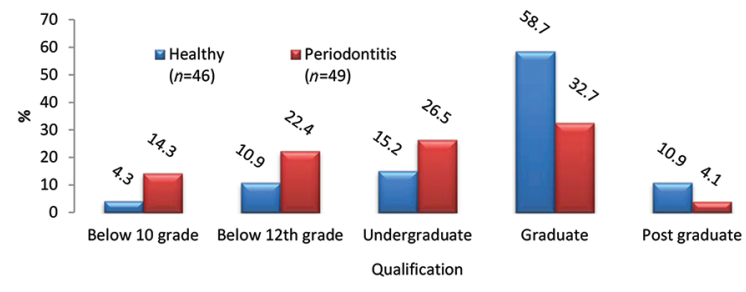

Fig. 2 Bar diagram depicting significant association of group II with respective qualification (32.7\%). 
Table 2 Mean comparison of osteocalcin concentrations and clinical parameters of both groups with severity of periodontitis

\begin{tabular}{|c|c|c|c|c|c|c|c|c|c|}
\hline \multirow[t]{3}{*}{ Parameters } & \multicolumn{8}{|c|}{ Periodontitis severity } & \multirow[t]{3}{*}{$p$-Value } \\
\hline & \multicolumn{2}{|c|}{$\begin{array}{l}\text { Normal } \\
(n=46)\end{array}$} & \multicolumn{2}{|c|}{$\begin{array}{l}\text { Mild } \\
(n=15)\end{array}$} & \multicolumn{2}{|c|}{$\begin{array}{l}\text { Moderate } \\
(n=19)\end{array}$} & \multicolumn{2}{|c|}{$\begin{array}{l}\text { Severe } \\
(n=15)\end{array}$} & \\
\hline & Mean & SD & Mean & SD & Mean & SD & Mean & SD & \\
\hline $\begin{array}{l}\text { Osteocalcin } \\
\text { concentration }\end{array}$ & 2.19 & 0.26 & 2.26 & 0.24 & 2.25 & 0.16 & 2.19 & 0.20 & 0.68 \\
\hline Absorbance (450 nm) & 0.13 & 0.32 & 0.09 & 0.03 & 0.09 & 0.02 & 0.08 & 0.02 & 0.83 \\
\hline Mean PPD & 1.28 & 0.59 & 2.67 & 0.49 & 4.00 & 0.94 & 6.27 & 0.46 & $<0.01^{\mathrm{a}}$ \\
\hline Mean BOP & 15.98 & 11.24 & 40.33 & 23.34 & 27.63 & 12.58 & 35.67 & 12.37 & $<0.01^{\mathrm{a}}$ \\
\hline CAL & - & - & 2.00 & 0.01 & 3.89 & 0.32 & 6.20 & 1.01 & $<0.01^{\mathrm{a}}$ \\
\hline Plaque index & 0.75 & 0.66 & 1.84 & 0.52 & 2.00 & 0.33 & 2.27 & 0.59 & $<0.01^{\mathrm{a}}$ \\
\hline
\end{tabular}

Abbreviations: ANOVA, analysis of variance; BOP, bleeding on probing; CAL, clinical attachment level; PPD, periodontal pocket depth; SD, standard deviation.

${ }^{a} p$-Value $<0.05$ was considered significant using one way ANOVA.

Table 3 Correlation analyses of osteocalcin concentration and clinical parameters in both study groups

\begin{tabular}{|l|l|l|l|l|l|l|l|l|l|}
\hline \multicolumn{2}{|c|}{ Parameters } & $\begin{array}{l}\text { Osteocalcin } \\
\text { concentration }\end{array}$ & Severity & CAL & $\begin{array}{l}\text { Plaque } \\
\text { index }\end{array}$ & $\begin{array}{l}\text { Mean } \\
\text { PPD }\end{array}$ & $\begin{array}{l}\text { Mean } \\
\text { BOP }\end{array}$ & Mobile teeth & Missing \\
\hline $\begin{array}{l}\text { Osteocalcin } \\
\text { concentration }\end{array}$ & $r$-Value & 1 & & & & & & & \\
\hline & $p$-Value & - & & & & & & & \\
\hline Severity & $r$-Value & 0.16 & 1 & & & & & & \\
\hline & $p$-Value & 0.13 & & & & & & & \\
\hline CAL & $r$-Value & 0.15 & 0.99 & 1 & & & & & \\
\hline & $p$-Value & 0.14 & $<0.01^{a}$ & & & & & & \\
\hline Plaque index & $r$-Value & 0.19 & 0.77 & 0.76 & 1 & & & & \\
\hline & $p$-Value & 0.07 & $<0.01^{a}$ & $<0.01^{\mathrm{a}}$ & & & & & \\
\hline Mean PPD & $r$-Value & 0.17 & 0.92 & 0.92 & 0.73 & 1 & & & \\
\hline & $p$-Value & 0.111 & $<0.01^{\mathrm{a}}$ & $<0.01^{\mathrm{a}}$ & $<0.01^{\mathrm{a}}$ & & & & \\
\hline Mean BOP & $r$-Value & 0.22 & 0.55 & 0.54 & 0.64 & 0.58 & 1 & & \\
\hline & $p$-Value & 0.06 & $<0.01^{\mathrm{a}}$ & $<0.01^{\mathrm{a}}$ & $<0.01^{\mathrm{a}}$ & $<0.01^{\mathrm{a}}$ & & & \\
\hline $\begin{array}{l}\text { Absorbance } \\
(450 \mathrm{~nm})\end{array}$ & $r$-Value & 1 & 0.16 & 0.15 & 0.19 & 0.17 & 0.22 & 0.17 & 0.13 \\
\hline & $p$-Value & $<0.01^{\mathrm{a}}$ & 0.13 & 0.14 & 0.07 & 0.1 & $0.03^{\mathrm{a}}$ & 0.10 & 0.20 \\
\hline
\end{tabular}

Abbreviations: BOP, bleeding on probing; CAL, clinical attachment level; PPD, periodontal pocket depth. ${ }^{a} p$-Value $<0.05$ considered significant for Spearman's rank correlation.

significant increase in mean values of PPD, BOP, CAL, and PI was observed concerning increasing in severity of periodontitis $(p<0.01)$. The mean of PPD was highest in severe periodontitis group $(6.27 \pm 0.46)$ and lowest in healthy individuals (1.28 \pm 0.59$)$, whereas mean PPD of mild $(2.67 \pm 0.49)$ was higher lower than moderate periodontitis group ( $4.00 \pm 0.94)$. Similarly, in group II the mean of CAL was highest in severe periodontitis group $(6.2 \pm 1.01)$ and lowest in mild periodontitis group $(2.0 \pm 0.01)$, respectively.

- Table 3 refers to the results of the nonparametric Spearman rank correlation test applied to assess the correlation of osteocalcin with periodontitis severity. A highly significant correlation was found between CAL, PPD, PI, and BOP and periodontitis $(p<0.01)$. Positive correlation of 99, 92, 77, and $55 \%$ was observed between disease severity and CAL, PPD, PI, and BOP, respectively. However, conversely CAL $(p=0.14)$, PI $(p=0.07)$, BOP $(p=0.06)$, and PPD $(p=0.11)$ were insignificantly correlated with osteocalcin levels. Furthermore, a nonsignificant correlation was found between osteocalcin and periodontitis severity $(p=0.13)$ indicating that osteocalcin levels might be independent of disease severity.

- Table 4 depicts results of chi-squared test to assess the association of periodontitis severity with bone loss. Nearly no bone loss was seen in majority (91.3\%) of healthy individuals, whereas highly significant bone loss was observed in $84.2 \%$ of moderate-to-severe periodontitis patients $(p<0.01)$ 
Table 4 Association of periodontitis severity with bone loss and radiograph

\begin{tabular}{|c|c|c|c|c|c|c|c|c|c|c|}
\hline \multirow{3}{*}{\multicolumn{2}{|c|}{ Parameters }} & \multicolumn{8}{|c|}{ Periodontitis severity } & \multirow[t]{3}{*}{$p$-Value } \\
\hline & & \multicolumn{2}{|c|}{ Normal $(n=46)$} & \multicolumn{2}{|c|}{ Mild $(n=15)$} & \multicolumn{2}{|c|}{$\begin{array}{l}\text { Moderate } \\
(n=19)\end{array}$} & \multicolumn{2}{|c|}{$\begin{array}{l}\text { Severe }(n \\
=15)\end{array}$} & \\
\hline & & $N$ & $\%$ & $N$ & $\%$ & $N$ & $\%$ & $N$ & $\%$ & \\
\hline \multirow[t]{5}{*}{ Bone loss } & No bone loss & 42 & 91.3 & - & - & - & - & - & - & \multirow[t]{5}{*}{$<0.01^{\mathrm{a}}$} \\
\hline & Less than 30 & 4 & 8.7 & 15 & 100 & - & - & - & - & \\
\hline & $30-50$ & - & - & - & - & 16 & 84.2 & - & - & \\
\hline & Nearly 50 & - & - & - & - & 3 & 15.8 & - & - & \\
\hline & More than 50 & - & - & - & - & - & - & 15 & 100 & \\
\hline \multirow[t]{2}{*}{ Radiograph } & Yes & 20 & 43.5 & 10 & 66.7 & 5 & 26.3 & 6 & 40 & \multirow[t]{2}{*}{0.13} \\
\hline & No & 26 & 56.5 & 5 & 33.3 & 14 & 73.7 & 9 & 60 & \\
\hline
\end{tabular}

${ }^{a} p$-Value $<0.05$ was considered significant using Pearson chi-squared test.

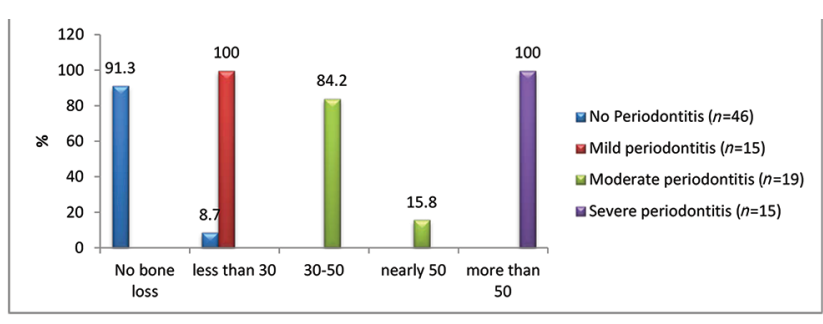

Fig. 3 Bar diagram illustrating association of group I and group II participants with bone loss. No bone loss was observed in $91.3 \%$ of healthy individuals, whereas $84.2 \%$ of bone loss was seen in moderate-to-severe periodontitis group.

( - Fig. 3). The bone loss scores were not statistically significant for radiographs $(p=0.13)$.

\section{Discussion}

In this study, we investigated levels of osteocalcin in the saliva of healthy and periodontitis patients and correlated them with disease severity. The mean age of the study participants was 31 to 45 years that coincided with the study conducted by Abdul Ameer and Ali where the mean sample age was also 30 to 50 years. ${ }^{25}$ The study subjects in the current study were predominantly male that is in parallel with a cross-sectional study done by $\mathrm{Ng}$ et al regarding evaluation of salivary bone markers in periodontitis, in which greater proportion of study population was males $(n=56)$ as compared with females $(n=42) .{ }^{26}$ The results of the present study showed significant association of qualification (graduates) with periodontitis group $(p<0.02)$ which is in line with the results of the study done by Hakeem and Sabbah, where also significant association of qualification was observed with periodontitis $(p<0.001){ }^{27}$

Our results revealed nonsignificant correlation of saliva osteocalcin with periodontitis $(p=0.13)$. Though all four clinical parameters were positively and significantly correlated with the periodontal status of the patients $(p<0.01)$, the saliva osteocalcin levels showed insignificant correlation with CAL, PPD, BOP, and PI having $p$-values of 0.14, 0.07, 0.06, and 0.11 , respectively.
The findings of our study are in consistent with other studies. In 2005, Bullon et al assessed saliva, serum, and gingival crevicular fluid (GCF) osteocalcin levels among healthy and periodontitis patients. No significant correlation was observed of PPD ( $p=0.11)$, PI $(p=0.41)$, and BOP ( $p=0.19$ ) with osteocalcin levels. ${ }^{28}$ In 1999, Lee evaluated levels of osteocalcin in adult periodontitis patients, and no statistically significant differences were reported between osteocalcin levels at diseased and healthy sites $(p=0.21)$. It was concluded that osteocalcin levels act as poor indicator of disease status. ${ }^{29}$ In another case-control study done by Ozçaka et al, nonsignificant correlation was seen between saliva osteocalcin and periodontal disease progression and severity $(p>0.05)$. Moreover, the controls exhibited markedly reduced values of all clinical periodontal measurements $(p<0.001)$. Hence, it was concluded that levels of osteocalcin might not provide possible information regarding periodontal disease severity and progression. ${ }^{30}$ Wilson et al in his case-control study analyzed osteocalcin, NTx, and albumin levels of 14 untreated periodontitis patients using ELISA technique. The results were interpreted as ratios OC/NTx ratio determined relative bone turnover, and OC/Alb or NTx/ Alb was used to determine osteocalcin or NTx synthesis. No association was found between osteocalcin levels and periodontitis. ${ }^{31}$ Becerik et al reported decreased or unchanged osteocalcin levels in GCF of chronic and aggressive periodontitis patients. These decreased or negligible levels of osteocalcin may be due to the inability of osteocalcin to differentiate sites with active alveolar bone loss and others in a bone loss arrest state. This theory pointed toward that the concept of abnormal bone turnover activity occurring in periodontitis. ${ }^{21}$

Contrary to our results, Betsy et al found elevated concentrations of salivary osteocalcin in periodontitis subjects as compared with controls. Furthermore, significant positive correlations of osteocalcin were present with BOP, PPD, and bone loss $(p<0.05){ }^{32}$ Similarly, Ng et al reported significant association of saliva osteocalcin with markers of alveolar bone loss $(p<0.001) .{ }^{26}$ Bullon et $\mathrm{al}^{33}$ in 2007 assessed saliva osteocalcin of 39 postmenopausal women and compared them with periodontal treatment outcome of 
patients. A significant decrease in CAL and disease severity was observed with low saliva osteocalcin after first appointment. However, this study was conducted on patients with already low bone density and altered bone metabolism that could possibly affect actual saliva osteocalcin levels due to periodontitis. The contrast between these results and our findings can be explained due to sampling bias. Sample selection of the current study was based on exclusion of confounding factors such as smoking, use of tobacco/betel nut, and individuals with any systemic or metabolic disorders. Due to bone remodeling process occurring at multiple skeletal bone resorption sites, the saliva osteocalcin concentrations are unlikely to reflect data peculiarly related to periodontal disease and the actual role of osteocalcin in disease severity would be masked.

Osteocalcin is considered as an established bone turnover marker in serum that maintains hemostasis during bone remodeling. In periodontitis, it is considered as a marker of bone formation where bone resorption rate is greater than bone formation due to activation of proinflammatory mediators and immune cells. However, in light of above-mentioned studies and data of the current study, it is concluded that decreased or negligible difference in saliva osteocalcin levels of both healthy and periodontitis group may not play predictive role in determining periodontal disease progression and severity.

However, the results of our study should be considered in light of two significant limitations. First, due to the small sample size of our study design, the findings could not be generalized to a larger population and are unable to find a cause-effect relationship. Second, the overall number of studies available in the literature relevant to the role of saliva osteocalcin in periodontitis is scanty. To overcome these limitations and validate our results, additional longitudinal and clinical-trial studies with larger sample size are required to determine actual levels of saliva osteocalcin in periodontitis as well elucidate its role if any in disease pathogenesis, progression, or in any other forms of periodontal disease.

\section{Conclusion}

The statistical data of our study demonstrated that saliva osteocalcin had a weak nonsignificant correlation with periodontitis. Though osteocalcin has been widely accepted as a bone turnover marker in serum, but low levels of osteocalcin in saliva of periodontitis patients indicate toward its poor role in disease progression and severity. Nevertheless, with the advances made in the field of salivary diagnostics, there is an unmet need for implementation of further clinical studies to determine the true role of osteocalcin in periodontitis. To achieve this goal, analysis of multiple biomarkers belonging to proteomic, genomic, microbial, and transcriptomics branches is favorable instead of a single biomarker alone. This multianalytical approach will lead to better diagnosis, prognosis, and treatment outcomes of periodontitis patients.

\section{Funding}

None.

\section{Conflict of Interest}

None declared.

\section{Acknowledgments}

The auhtors would like to thank Mr. Moazzam, Research Officer, Ziauddin University, for providing his expertise and technical skills while carrying out relevant laboratory procedures. We would also like to express our gratitude for all our colleagues for sharing their pearls of wisdom that much helped in improving the quality of our article.

\section{References}

1 Hajishengallis G. Periodontitis: from microbial immune subversion to systemic inflammation. Nat Rev Immunol 2015;15(1):30-44

2 Llanos AH, Silva CGB, Ichimura KT, et al. Impact of aggressive periodontitis and chronic periodontitis on oral health-related quality of life. Braz Oral Res 2018;32:e00610.1590/18073107bor-2018.vol32.0006

3 Nazir MA. Prevalence of periodontal disease, its association with systemic diseases and prevention. Int J Health Sci (Qassim) 2017;11(2):72-80

4 Meyle J, Chapple I. Molecular aspects of the pathogenesis of periodontitis. Periodontol 2000 2015;69(1):7-17

5 Tonetti MS, Jepsen S, Jin L, Otomo-Corgel J. Impact of the global burden of periodontal diseases on health, nutrition and wellbeing of mankind: a call for global action. J Clin Periodontol 2017;44(5):456-462

6 Damgaard C, Holmstrup P, Van Dyke TE, Nielsen CH. The complement system and its role in the pathogenesis of periodontitis: current concepts. J Periodontal Res 2015;50(3):283-293

7 Eke PI, Dye BA, Wei L, et al. Update on prevalence of periodontitis in adults in the United States: NHANES 2009 to 2012. J Periodontol 2015;86(5):611-622

8 Papapanou PN, Susin C. Periodontitis epidemiology: is periodontitis under-recognized, over-diagnosed, or both.? Periodontol 2000 2017;75(1):45-51

9 Corbet EF, Leung WK. Epidemiology of periodontitis in the Asia and Oceania regions. Periodontol 2000 2011;56(1):25-64

10 Bokhari SA, Suhail AM, Malik AR, Imran MF. Periodontal disease status and associated risk factors in patients attending a dental teaching hospital in Rawalpindi, Pakistan. J Indian Soc Periodontol 2015;19(6):678-682

11 Sahibzada HA, Khurshid Z, Khan RS, et al. Salivary IL-8, IL-6 and TNF- $\alpha$ as potential diagnostic biomarkers for oral cancer. Diagnostics (Basel) 2017;7(2):2110.3390/diagnostics7020021

12 Khurshid Z. Salivary point-of-care technology. Eur J Dent 2018;12(1):1-2

13 Khan RS, Khurshid Z, Yahya Ibrahim Asiri F. Advancing pointof-care (PoC) testing using human saliva as liquid biopsy. Diagnostics (Basel) 2017;7(3):39

14 Abdul Rehman S, Khurshid Z, Hussain Niazi F, et al. Role of salivary biomarkers in detection of cardiovascular diseases (CVD) Proteomes 2017:5(3):21

15 Khurshid Z, Zafar M, Khan E, Mali M, Latif M. Human saliva can be a diagnostic tool for Zika virus detection. J Infect Public Health 2019;12(5):601-604

16 Jaedicke KM, Preshaw PM, Taylor JJ. Salivary cytokines as biomarkers of periodontal diseases. Periodontol 2000 2016;70(1):164-183

17 Javaid MA, Ahmed AS, Durand R, Tran SD. Saliva as a diagnostic tool for oral and systemic diseases. J Oral Biol Craniofac Res 2016;6(1):66-75

18 Gursoy UK, Liukkonen J, Jula A, et al. Associations between salivary bone metabolism markers and periodontal breakdown. J Periodontol 2016;87(4):367-375 
19 Schulze-Späte U, Turner R, Wang Y, et al. Relationship of bone metabolism biomarkers and periodontal disease: the osteoporotic fractures in men (MrOS) study. J Clin Endocrinol Metab 2015;100(6):2425-2433

20 Hienz SA, Paliwal S, Ivanovski S. Mechanisms of bone resorption in periodontitis. J Immunol Res 2015;2015:61548610.115 5/2015/615486

21 Becerik S, Afacan B, Oztürk VÖ, Atmaca H, Emingil G. Gingival crevicular fluid calprotectin, osteocalcin and cross-linked $\mathrm{N}$-terminal telopeptid levels in health and different periodontal diseases. Dis Markers 2011;31(6):343-352

22 Kassebaum NJ, Bernabé E, Dahiya M, Bhandari B, Murray CJL, Marcenes W. Global burden of severe periodontitis in 19902010: a systematic review and meta-regression. J Dent Res 2014;93(11):1045-1053

23 Caton JG, Armitage G, Berglundh T, et al. A new classification scheme for periodontal and peri-implant diseases and conditions - introduction and key changes from the 1999 classification. J Periodontol 2018;89(Suppl 1) :S1-S8

24 Bellagambi FG, Lomonaco T, Salvo P, et al. Saliva sampling: methods and devices. An overview. TrAC Trends Anal Chem 2020;124:115781

25 Abdul Ameer LA, Ali BG. Effects of light smoking on salivary levels of alkaline phosphatase and osteocalcin in chronic periodontitis patients. J Baghdad Coll Dent 2015;27(2):110-114

26 Ng PYB, Donley M, Hausmann E, Hutson AD, Rossomando EF, Scannapieco FA. Candidate salivary biomarkers associated with alveolar bone loss: cross-sectional and in vitro studies. FEMS Immunol Med Microbiol 2007;49(2):252-260
27 Hakeem FF, Sabbah W. Is there socioeconomic inequality in periodontal disease among adults with optimal behaviours. Acta Odontol Scand 2019;77(5):400-407

28 Bullon P, Goberna B, Guerrero JM, Segura JJ, Perez-Cano R, Martinez-Sahuquillo A. Serum, saliva, and gingival crevicular fluid osteocalcin: their relation to periodontal status and bone mineral density in postmenopausal women. J Periodontol 2005;76(4):513-519

29 Lee AJ, Walsh TF, Hodges SJ, Rawlinson A. Gingival crevicular fluid osteocalcin in adult periodontitis. J Clin Periodontol 1999;26(4):252-256

30 Ozçaka O, Nalbantsoy A, Köse T, Buduneli N. Plasma osteoprotegerin levels are decreased in smoker chronic periodontitis patients. Aust Dent J 2010;55(4):405-410

31 Wilson AN, Schmid MJ, Marx DB, Reinhardt RA. Bone turnover markers in serum and periodontal microenvironments. J Periodontal Res 2003;38(4):355-361

32 Betsy J, Ahmed JM, Mohasin AK. Mohammed A, Nabeeh A A. Diagnostic accuracy of salivary biomarkers of bone turnover in identifying patients with periodontitis in a Saudi Arabian population. J Dent Sci 2019;14(3):269-276

33 Bullon P, Chandler L, Segura E, J J., Perez CR, Martinez SA. Osteocalcin in serum, saliva and gingival crevicular fluid: their relation with periodontal treatment outcome in postmenopausal women. Medicina Oral, Patología Oral y Cirugía Bucal (Internet), 2017;12(3):193-197 